\title{
Observer-Based Bilinear Control of First-Order Hyperbolic PDEs: Application to the Solar Collector
}

\author{
Sarah Mechhoud and Taous-Meriem Laleg-Kirati
}

\begin{abstract}
In this paper, we investigate the problem of bilinear control of a solar collector plant using the available boundary and solar irradiance measurements. The solar collector is described by a first-order 1D hyperbolic partial differential equation where the pump volumetric flow rate acts as the plant control input. By combining a boundary state observer and an internal energy-based control law, a nonlinear observer based feedback controller is proposed. With a feed-forward control term, the effect of the solar radiation is cancelled. Using the Lyapunov approach we prove that the proposed control guarantees the global exponential stability of both the plant and the tracking error. Simulation results are provided to illustrate the performance of the proposed method.
\end{abstract}

\section{INTRODUCTION}

A solar collector is a spatially distributed engineering system which aims at collecting and generating energy from solar radiation. With the constant world increasing demand on energy and the urgent need of new environment-friendly energy resources, solar collector plants offer a clean and inexhaustible energy technology. Thanks to their long-term benefits, they are able to replace fossil fuels and enhance sustainability by exploiting the radiant light and heat of the sun. A solar parabolic through consists of a parabolic surface which focuses the solar radiations entering its plane of symmetry onto the focal line where a receiver tube is placed. In this tube a fluid (oil in general) absorbs this concentrated heat while it is transported. The heat-absorbing fluid (oil) is then piped to a heat engine to feed a power generating system, a desalination plant or to an oil-cooling system [1].

Using the energy balance principle, the solar collector is generally described by a pure heat transport equation where only the advection phenomenon occurs. The pump volumetric flow rate is the control input, the outlet temperature and the solar irradiance are the available measurements ([2] and references therein). The last years have noticed extensive studies on control strategies devoted to solar collectors. A survey on automatic control of the solar collector outlet temperature can be found in [1], where various control techniques ranging from simple PID controllers to more advanced strategies such as fuzzy logic, neural network and hierarchical controllers were proposed. However, all these methods were based on a discrete model of the solar collector neglecting its distributed nature. It has been pointed out in

S. Mechhoud and T. M Laleg-Kirati are with Computer, Electrical and Mathematical Sciences and Engineering Division, King Abdullah university of science and technology, Thuwal 23955-6900, Saudi Arabia, email: \{sarra.mechhoud, taousmeriem.laleg\}@kaust.edu.sa
[3] that unlike parabolic PDEs, almost all the eigenmodes of the spatial differential operator of a hyperbolic PDE carry nearly the same amount of energy. Consequently, there is no gap between the fast modes (transient behavior) and the slow modes (main dynamics). This implies that an infinite number of modes is required to describe the PDE dynamics. Furthermore, in [4], it was reported that especially for hyperbolic PDEs, the approximate dynamics are very sensitive to the nature of the discretization scheme often leading to he loss of the most important dynamics i.e. if the discretization scheme is not chosen carefully (which is not an easy task), the dynamics of the resulted reduced-order model may differ considerably from those of the original PDE. Thereby, it is difficult to catch the main dynamics of the solar collector model using a finite order discretized model. In [5] a new control strategy based on the passivity theory was proposed. Using the internal energy a distributed stable and robust control law relying on feedback from the distributed collector fields internal energy was introduced. A feedforward from the measured solar radiation was also incorporated for disturbance rejection performance. However, the presented control strategy relied on a distributed sensing, yet in the implementation section, the authors referred to the implementation of an observer in the open loop without further explanations.

There have been extensive studies in the last decade on boundary adaptive observer design and control techniques of first-order hyperbolic systems ([6], [7], [8], [9], [10], [11] and references therein). Nevertheless, late-lumping bilinear control of first-order bilinear PDEs has not attracted much attention of the community. In [12], a control technique of first-order hyperbolic PDEs based on the characteristic index (relative degree) was proposed. This method is widely applied for heat exchangers, fixed-bed reactors, plug-flow reactors, etc ([13] and references therein). An LQ-controller for a class of hyperbolic PDEs can be found in [14] and references therein.

In this work, inspired from [5], we aim at developing a new control technique which takes into considerations the practical constraints on the volumetric flow rate while ensuring the tracking of the reference setpoint. Like in [1] (and references therein) a feedforward term is included to cancel the variations of the solar radiations. Unlike [5], we show that our control strategy satisfies the limitations on the flow rate and guarantees the point-wise convergence of the interior and outlet tracking errors by extending the separation principle to the class of bilinear first-order hyperbolic PDEs that describe the solar collector. The proposed control law is based on a 
passivity-like principle and a boundary state observer. The stability of the closed-loop system is investigated in the Lyapunov-approach.

The method developed in this work may find application in various systems that share the same description including spray driers, high way traffic, heat exchangers and plug-flow reactors.

This paper is organized as follows. In Section 2, the solar collector model and the control problem are formulated. The control law in case of a fully distributed sensing is detailed in Section 3. In Section 4, the boundary state observer is developed. The convergence of the combination of the control strategy and the boundary state observer in the closed-loop is presented in Section 5. Simulation results are depicted in Section 6.

\section{PRoblem FORMULATION}

Using energy balance laws, the solar collector field can be described by the heat transport of the circulating fluid. Moreover, if the heat losses and the conductivity of the tube are neglected, the following model is obtained [2] (and references therein):

$$
\left\{\begin{array}{l}
\left.\left.\frac{\partial T}{\partial t}=-q(t) \frac{\partial T}{\partial x}+f(t), \quad x \in\right] 0,1\right], \quad t \geq 0 \\
T(x=0, t)=g(t), \quad t \geq 0 \\
\left.\left.T(x, t=0)=T_{0}(x), \quad x \in\right] 0,1\right]
\end{array}\right.
$$

with:

$$
f(t)=\frac{\eta_{0} G}{\rho c S} I(t)
$$

and where $T(x, t)$ is the fluid temperature, $q(t)$ is the fluid pump volumetric flow, $\eta_{0}$ is the mirror optical efficiency, $G$ is the tube aperture, $\rho$ is the fluid density, $c$ is the specific heat capacity, $S$ is the cross-sectional area and $I(t)$ is the solar radiation. The normalized space and time variables are denoted by $x$ and $t$ respectively. The second and third equations in system (1) describe the boundary and initial conditions respectively. They fulfil the following zero-order compatibility condition:

$$
g(t=0)=T_{0}(x=0) .
$$

The output equation is given by:

$$
y(t)=T(1, t),
$$

referring to measurements of the temperature at the tube outlet where $x=1$. We admit the following assumptions:

\section{Assumptions:}

(i) $q \in Q_{q}=\left\{q \in C^{1}(0, t): 0 \leq q_{\min } \leq q(t) \leq q_{\max }, \forall t \geq 0\right\}$,

(ii) $f \in Q_{f}=\left\{f \in L^{2}(0, t): f(t)>0, \forall t \geq 0\right\}$,

(iii) $T_{0} \in H^{1}(0,1)$.

These assumptions are compatible with the physical characteristics of $q(t)$ and $I(t)$, since the flow rate $q(t)$ is lower bounded to ensure good operation conditions of the solar plant and upper bounded due to the physical limitations of the fluid pump [2]. The solar radiation $I(t)$ is by definition positive.

They also ensure the well-posedness of the PDE given by (1). In fact, let $A(t)$ denotes the operator given by:

$$
A(t)=-q(t) \frac{\partial}{\partial x}
$$

The domain of the operator $A(t)$ is independent of the time and is given by:

$$
D(A(t))=H^{1}(0,1):=\left\{h \in L_{2}(0,1): \frac{\partial h}{\partial x} \in L_{2}(0,1)\right\},
$$

where $L_{2}$ is the usual space of square integrable function endowed with the standard norm $\|.\|_{2}$.

If assumption $(i)$ is satisfied, the operator $A(t)$ is an infinitesimal generator of a $C_{0}$-semigroup of contraction and the family $\{A(t)\}_{t \geq 0}$ is stable ([15], chapter 4). This statement and assumptions (ii) and (iii) assert that the PDE (1) admits a unique regular $H^{1}$-valued solution $\left(T \in C^{1}\left(0, t ; H^{1}(0,1)\right)\right)$ [15]. Moreover, if $q(t) \in L^{\infty}(0, t)$ and $T_{0} \in L_{2}(0,1)$ then PDE (1) has a unique weak solution $T \in C^{0}\left(0 ; t ; L_{2}(0,1)\right)[15]$.

Let $T^{*}(x, t)$ be the desired temperature profile. The objective of this work is to find a control input $q_{\min }<q(t)<q_{\max }$ that drives the average tracking error $\delta(t)$ defined by:

$$
\delta(t)=\int_{0}^{1}\left(T^{*}(x, t)-T(x, t)\right) d x, \quad t \geq 0,
$$

to zero (in a predefined sense) while assuming that the boundary condition $T(x=0, t)$ is known and the outlet temperature $T(x=1, t)$ and the solar radiation $I(t)$ are measured.

Proposition 2.1: The tracking error $\delta(t)$ in (6) is positive if:

$$
T^{*}(x, t=0) \geq T(x, t=0)
$$

where $T^{*}(x, t=0)$ is the initial condition of the target system generating the desired temperature profile $T^{*}(x, t)$ and $T(x, t=0)$ is the initial condition of system (1).

Proof: The target system is defined by PDE (1) by choosing the initial condition to be $T^{*}(x, t=0)$ while the volumetric flow rate $q(t)$ is replaced by the desired one $q^{*}$ satisfying assumption (i).

Using the method of characteristics, the analytic solution of (1) is given by:

$T(x, t)=\left\{\begin{array}{l}\left.T_{0}\left(x-\int_{0}^{t} q(\tau) d \tau\right)+\int_{0}^{t} f(\tau) d \tau, \quad \text { if } x \geq \int_{0}^{t} q(\tau)\right) d \tau \\ \left.g(t-\gamma)+\int_{0}^{\gamma} f(\tau) d \tau, \quad \text { if } x<\int_{0}^{t} q(\tau)\right) d \tau\end{array}\right.$

where $\gamma=\int_{0}^{x} \frac{d \alpha}{q(r(\alpha))}$ and $r(x)$ is the inverse of the characteristic curve equation $x(t)$, for more details on the 
method of characteristics see [16], chapter 2. From the analytic solution $\delta(t)$ is given by:

$$
\delta(t)=\int_{0}^{1}\left(T_{0}^{*}\left(x-\int_{0}^{t} q^{*}(\tau) d \tau\right)-T_{0}\left(x-\int_{0}^{t} q(\tau) d \tau\right)\right) d x
$$

and thus if condition given in (7) is satisfied the average tracking error is positive.

Remark 2.1: The convergence towards zero and the positivity of $\delta(t)$ guarantee the point-wise convergence of the distributed tracking error $\left(T^{*}(x, t)-T(x, t)\right)$ and consequently the convergence of the outlet tracking error

$\left(T^{*}(x=1, t)-T(x=1, t)\right)$.

$$
\delta(t) \longrightarrow 0 \Longrightarrow\left(T^{*}(x=1, t)-T(x=1, t)\right) \longrightarrow 0 .
$$

Throughout this work, we assume that the target profile $T^{*}$ is constructed such that condition (7) is fulfilled.

\section{ENERGY-BASED CONTROL}

The energy-based control for solar collector plants was first proposed by [5] in 2002, where the core idea was to use the internal energy as the key controlled variable. The result is a nonlinear PID controller coupled with a feedforward term to cancel the effect of the solar radiation.

In this section, we assume first that a distributed sensing of the temperature profile $T(x, t)$ is available and we design a control law $q(t)$ which drives the average tracking error $\delta(t)$ towards zero.

Inspired by the work of [5], we consider the average temperature $U(t)$ over the space as the controlled variable:

$$
U(t)=\int_{0}^{1} T(x, t) d x
$$

Theorem 3.1: Consider the PDE (1) subject to assumptions (i)-(iii). Let the control input $q(t)$ be given by:

$$
q(t)=\frac{1}{(T(1, t)-T(0, t))}\left[f(t)-\int_{0}^{1} \frac{\partial T^{*}}{\partial t} d x-k_{p} \delta(t)\right]
$$

then the average tracking error $\delta(t)$ converges exponentially to zero, i.e:

$$
\exists M, \alpha>0:|\delta(t)| \leq M|\delta(0)| \mathrm{e}^{-\alpha t}
$$

Proof: Let $V_{1}$ given in (12) be a Lyapunov candidate function:

$$
V_{1}(\delta(t))=\frac{1}{2} \delta(t)^{2}
$$

Its derivative is given by:

$$
\dot{V}_{1}(\delta(t))=\delta(t) \int_{0}^{1}\left\{q(t) \frac{\partial T}{\partial x}-f(t)+\frac{\partial T}{\partial t}\right\} d x
$$

By replacing $q(t)$ by its formula given in (11) into (14), we obtain:

$$
\dot{V}_{1}(\delta(t))=-k_{p} \delta(t)^{2}
$$

and thus

$$
V(t) \leq V(0) \mathrm{e}^{-k_{p} t}
$$

and we conclude that using the control input $q(t)$ defined in (11), the average tracking error $\delta(t)$ converges globally and exponentially to zero. From Proposition 2.1 and Remark 2.1 we conclude that the outlet temperature tracking error $T^{*}(x=1, t)-T(x=1, t)$ converges exponentially to zero.

Remark 3.1: Since $T^{*}(x, t)$ is the output of the target model which has the same expression as PDE (1) and by replacing $q(t), T_{0}(x)$ by the desired $q^{*}(t), T_{0}^{*}(x)$, the control input $q(t)$ proposed in (11) can be written as:

$q(t)=\frac{1}{(T(1, t)-T(0, t))}\left[q^{*}(t)\left(T^{*}(1, t)-T^{*}(0, t)\right)-k_{p} \delta(t)\right]$.

Since $\delta(t)$ converges exponentially to zero and is bounded, we see that if $q^{*}$ satisfies assumption (i) then even $q(t)$ given by (11) satisfies this assumption.

\section{BOUNDARY STATE OBSERVER DESIGN}

In the previous section, we have seen that using a distributed sensing $T(x, t)$ one can design an admissible control input that drives the tracking error (average and point-wise) towards zero exponentially. However, in practice a distributed sensing does not exist. The only available measurements are those of the solar radiation $I(t)$ using a pyrheliometer [17] (and references therein) and of the outlet temperature $T(1, t)$. To overcome this limitation, we first develop a state observer based on the available measurements and prove its convergence and then in the next section we will demonstrate that the coupling between this state observer and the control law given by (11) leads to the exponential convergence of the tracking error.

Let the state observer be given by:

$$
\left\{\begin{array}{l}
\left.\left.\frac{\partial \hat{T}}{\partial t}=-q(t) \frac{\partial \hat{T}}{\partial x}+f(t), \quad x \in\right] 0,1\right], t \geq 0 \\
\hat{T}(0, t)=g(t)-k_{0} \Delta T(1, t), \\
\hat{T}(x, 0)=\hat{T}_{0}(x) \geq 0,
\end{array}\right.
$$

where $\Delta T(x, t)=\hat{T}(x, t)-T(x, t)$ is the state estimation error $\Delta T(1, t)$ is the correction term (output error). This state observer is a Luenberger-like observer, however, the correction term $\Delta T(1, t)$ is injected in the boundary $\hat{T}(0, t)$ rather than being inserted in the state equation.

The time evolution of the time derivative of $\Delta T$ is given by:

$$
\left\{\begin{array}{l}
\frac{\partial \Delta T}{\partial t}=-q(t) \frac{\partial \Delta T}{\partial x}, \\
\Delta T(0, t)=-k_{0} \Delta T(1, t), \\
\Delta T(x, 0)=\hat{T}_{0}(x)-T_{0}(x) .
\end{array}\right.
$$

Proposition 4.1: If $0<\left|k_{0}\right| \leq \mathrm{e}^{\mu}$ where $\mu$ is a tuning positive parameter, the state observer introduced in (16) is an exponential boundary observer.

Proof: Let us define the following Lyapunov candidate function $V_{2}$ which was first used in [18] to analyze the stability of linear symmetric hyperbolic systems:

$$
V_{2}(t)=\int_{0}^{1} \mathrm{e}^{-\mu}(\Delta T(x, t))^{2} d x
$$


The derivative of $V_{2}$ is written as:

$$
\begin{aligned}
\dot{V}_{2}(t) & =2 \int_{0}^{1} \mathrm{e}^{-\mu x} \Delta T(x, t) \frac{\partial \Delta T}{\partial t} d x \\
& =-q(t) \int_{0}^{1} \mathrm{e}^{-\mu x} \frac{\partial \Delta T^{2}}{\partial x} d x,
\end{aligned}
$$

Using integration by parts, we obtain:

$$
\begin{aligned}
\dot{V}_{2}(t) & =-q(t) \mu \int_{0}^{1} \mathrm{e}^{-\mu x} \Delta T(x, t)^{2} d x \\
& -q(t)\left[\mathrm{e}^{-\mu} \Delta T(1, t)^{2}-\Delta T(0, t)^{2}\right],
\end{aligned}
$$

By replacing the boundary condition of (16) in (20) we get:

$$
\begin{aligned}
\dot{V}_{2}(t) & =-q(t) \mu \int_{0}^{1} \mathrm{e}^{-\mu x} \Delta T(x, t)^{2} d x \\
& -q(t)\left(\mathrm{e}^{-\mu}-k_{0}^{2}\right) \Delta T(1, t)^{2} \\
& \leq-q_{\max } \mu V_{2}(t)
\end{aligned}
$$

and therefore we conclude that:

$$
V_{2}(t) \leq V_{2}(0) \mathrm{e}^{-\mu q_{\max } t}
$$

and from the equivalence between the norms $\|\cdot\|_{2, \mathrm{e}^{-\mu x}}$ and $\|\cdot\|_{2}$ we infer that:

$$
\|\Delta T(., t)\|_{2} \leq\|\Delta T(., 0)\|_{2} \mathrm{e}^{-q_{\max } \mu},
$$

which complete the proof.

\section{Stability of The ObSERVER-BASED CONTROL SYSTEM}

In this section, we prove the stability of the coupling of the state observer and the control input law defined in (11). Let $\hat{\delta}$ be the average difference between the observed state $\hat{T}$ and the desired temperature profile $T^{*}$ :

$$
\hat{\delta}(t)=\int_{0}^{1}\left(T^{*}(x, t)-\hat{T}(x, t)\right) d x .
$$

The time variation of $\hat{\delta}(t)$ is as follows:

$$
\dot{\hat{\delta}}(t)=q(t)[\hat{T}(1, t)-\hat{T}(0, t)]-f(t)+\int_{0}^{1} \frac{\partial T^{*}}{\partial t} d x .
$$

Theorem 5.1: Consider the PDE given by (1) subject to assumptions (i)-(iii). Let $\hat{T}$ be given by PDE (16) and let the control input $q(t)$ given by:

$$
q(t)=\frac{1}{(T(1, t)-T(0, t))}\left[f(t)-\int_{0}^{1} \frac{\partial T^{*}}{\partial t} d x-k_{p} \hat{\delta}(t)\right]
$$

ensures the exponential convergence of the tracking error $\delta(t)$ and the state estimation error $\Delta T(x, t)$.

Proof: The proof is based on Lyapunov theory. Let us define the following Lyapunov candidate function:

$$
V(t)=\frac{1}{2} \delta^{2}(t)+\int_{0}^{1} \mathrm{e}^{-\mu x}(\Delta T(x, t))^{2} d x .
$$

The tracking error $\delta(t)$ can be written as:

$$
\delta(t)=\hat{\delta}(t)+\int_{0}^{1} \Delta T(x, t) d x .
$$

Using (17) and (25), the derivative of (6) writes:

$$
\dot{\delta}(t)=q(t)[T(1, t)-T(0, t)]-f(t)+\int_{0}^{1} \frac{\partial T^{*}}{\partial t} d x .
$$

Substituting (26) in (29) gives:

$$
\dot{\delta}(t)=-k_{p} \hat{\delta}(t) .
$$

Thereby, the derivative of $V$ in (27) is given by:

$$
\begin{aligned}
\dot{V}(t) & =-k_{p} \hat{\delta}^{2}(t)-k_{p} \hat{\delta}(t) \int_{0}^{1} \Delta T(x, t) d x \\
& -q(t) \mu \int_{0}^{1} \mathrm{e}^{-\mu x} \Delta T^{2}(x, t) d x \\
& -q(t)\left(\mathrm{e}^{-\mu}-k_{0}^{2}\right) \Delta T^{2}(1, t) .
\end{aligned}
$$

Since $q(t)$ satisfies assumption (i), equation (32) becomes:

$$
\begin{aligned}
\dot{V}(t) \leq & -k_{p} \hat{\delta}^{2}(t)-k_{p} \hat{\delta}(t) \int_{0}^{1} \Delta T(x, t) d x \\
& -q_{\max } \mu \int_{0}^{1} \mathrm{e}^{-\mu x} \Delta T^{2}(x, t) d x,
\end{aligned}
$$

From (28) inequality (32) becomes:

$$
\begin{aligned}
\dot{V}(t) \leq & -k_{p} \delta^{2}(t)-k_{p} \int_{0}^{1}\left(\Delta T^{2}(x, t)\right)^{2} d x+2 k_{p} \mid \delta(t) \\
& \left|\int_{0}^{1}\right| \Delta T(x, t) \mid d x-k_{p} \hat{\delta}(t) \int_{0}^{1} \Delta T(x, t) d x \\
& -q_{\max } \mu \int_{0}^{1} \mathrm{e}^{-\mu x} \Delta T^{2}(x, t) d x, \\
\leq & -k_{p} \delta^{2}(t)-q_{\max } \mu\|\Delta T(x, t)\|_{2}^{2} \\
& +k_{p}(|\hat{\delta}(t)|+2|\delta(t)|) \int_{0}^{1}|\Delta T(x, t)| d x .
\end{aligned}
$$

Let $M=\min \left(k_{p}, q_{\max } \mu\right)$, then (33) can be written as:

$$
\dot{V}(t) \leq-M V(t)+k_{p}(|\hat{\delta}(t)|+2|\delta(t)|) \int_{0}^{1}|\Delta T(x, t)| d x .
$$

The state tracking error $\delta(t)$ and the estimated tracking error $\hat{\delta}(t)$ lie in compact spaces and thus are bounded, i.e:

$$
\exists \alpha_{1}, \alpha_{2}>0: \delta(t) \leq \alpha_{1}, \hat{\delta}(t) \leq \alpha_{2} .
$$

This allows us to write (34) as:

$$
\dot{V}(t) \leq-M V(t)+k_{p} \alpha \int_{0}^{t}|\Delta T(x, t)| d x .
$$


where $\alpha=\alpha_{1}+\alpha_{2}$. Let us denote the second member of inequality (36)'s right side by $\varepsilon(t)$ i.e.:

$$
\begin{aligned}
\varepsilon(t)= & k_{p} \alpha \int_{0}^{1}|\Delta T(x, t)| d x \\
& \leq k_{p} \alpha\|\Delta T(., t)\|_{2}^{2} \\
& \leq k_{p} \alpha\|\Delta T(., 0)\|_{2}^{2} \mathrm{e}^{-2 q_{\max } \mu t}, \\
& \leq k_{p} \alpha\|\Delta T(., 0)\|_{2}^{2} \mathrm{e}^{-2 M t},
\end{aligned}
$$

the second inequality is due to inequality (23) whereas the third one is due to the definition of $M$.

Taking into account the last inequality in (37) and using Gronwall's inequality in (36) we get:

$$
\begin{aligned}
\dot{V}(t) \leq & V(0) \mathrm{e}^{-M t}+\int_{0}^{t} \mathrm{e}^{-M(t-s)} \varepsilon(s) d s \\
& \leq V(0) \mathrm{e}^{-M t}+k_{p} \alpha \int_{0}^{t} \mathrm{e}^{-M(t-s)}\|\Delta T(., 0)\|_{2}^{2} \mathrm{e}^{-2 M s} d s \\
& \leq\left(V(0)+\frac{k_{p} \alpha}{M}\|\Delta T(., 0)\|_{2}^{2}\right) \mathrm{e}^{-M t},
\end{aligned}
$$

the last inequality is obtained by computing the integral term and at this stage we conclude the global exponential convergence of $\delta(t)$ and $\|\Delta T(., t)\|_{2}$.

Remark 5.1: The performance of the proposed control input in (26) can be improved by adding the integral and derivative actions i.e.:

$$
\begin{aligned}
q(t) & =\frac{1}{(T(1, t)-T(0, t))}\left[f(t)-\int_{0}^{1} \frac{\partial T^{*}}{\partial t} d x\right. \\
& \left.-k_{p}\left(\hat{\delta}(t)+\frac{1}{T_{i}} \int-0^{t} \hat{\delta}(\tau) d \tau+\frac{1}{T_{d}} \dot{\hat{\delta}}(t)\right)\right] .
\end{aligned}
$$

For the sake of clarity these terms were not added in the proof. However using the same steps as in the previous proof, we can demonstrate the convergence of the tracking error. The core idea is that all the terms multiplying the term $\int_{0}^{1} \Delta T(x, t) d x$ will vanish exponentially since they are bounded and the $L_{2}$ norm of the state estimation error $\Delta T$ is exponentially convergent.

\section{Simulation RESUlTS}

In this section, using the developed control strategy on the model of a solar collector, we seek the admissible control $q(t)$ able to drive the system from its initial condition to the reference temperature profile given in fig. 1 under given solar radiations. This profile is obtained from the following formula:

$$
T^{*}(x, t)=T^{*}(0, t)+x \times\left(T^{*}(1, t)-T^{*}(0, t)\right), \quad x \in[0,1],
$$

where the initial and boundary temperatures were fixed at $20^{\circ} \mathrm{C}$. These profiles were chosen based on the work of [2] and [5].

Using the control input given in (26)-(16) and starting from

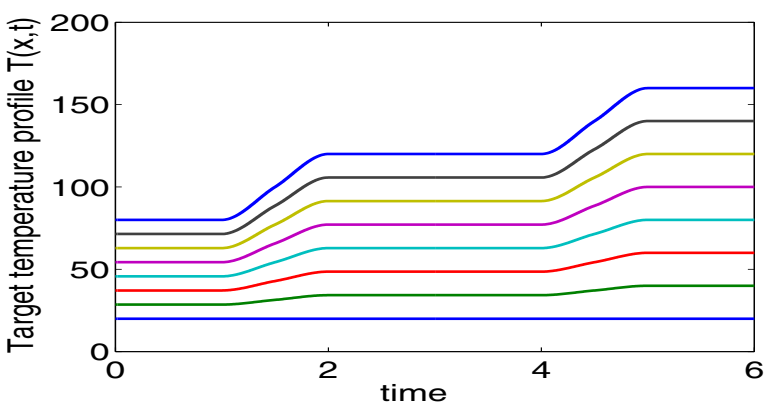

Fig. 1: Reference temperature profile $\delta(t)$

an initial condition which was fixed at $20^{\circ} \mathrm{C}$, the evolution of the average temperature tracking error $\delta(t)$ is given in fig. 2. This figure shows that $\delta(t)$ converges rapidly and in finite time. The profile of $q(t)$ is given in fig. 3. The decrease of the flow rate $q(t)$ correspond to the increase of $s$ the temperature which is physically coherent. The relative

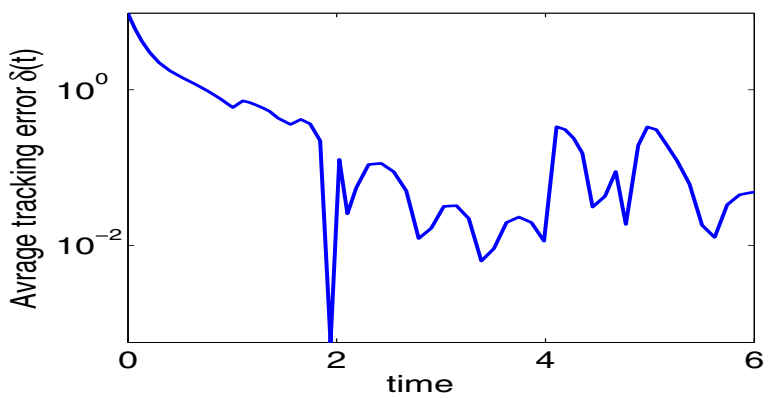

Fig. 2: Average tracking error $\delta(t)$

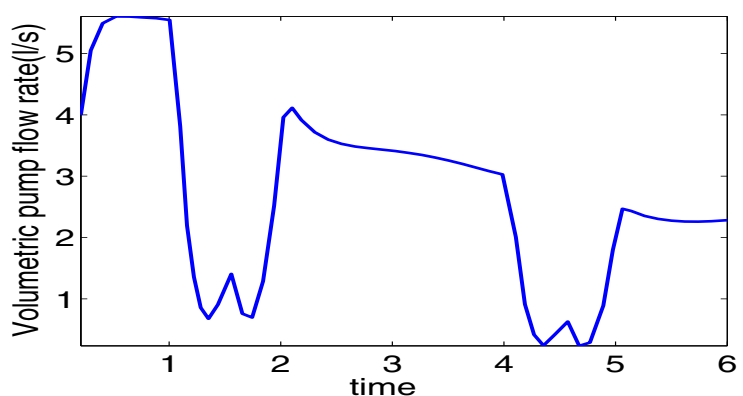

Fig. 3: Volumetric flow rate $q(t)$

state estimation error using the boundary observer given by (16) is depicted in fig. 4. The estimation error as supposed converges exponentially towards zero. From fig. 5, the outlet temperature relative tracking error $T^{*}(1, t)-T(1, t)$ is convergent which confirms the statement that the convergence of $\delta(t)$ induce the point-wise convergence of the temperature tracking profile.

These simulations were performed using the quadratic bsplines Galerkin method. 


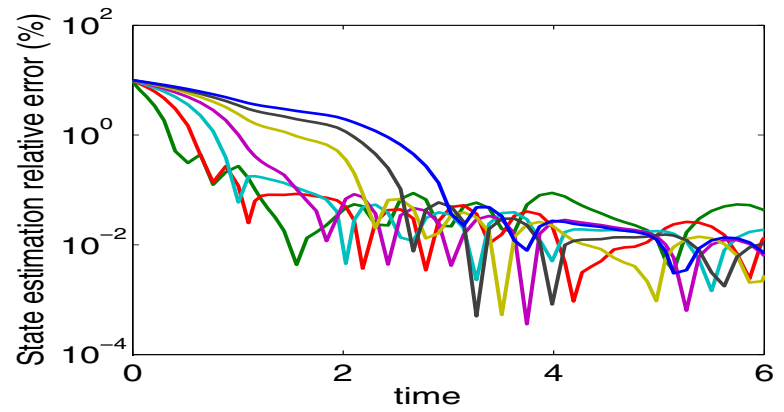

Fig. 4: Relative state estimation error $\Delta T(x, t)$

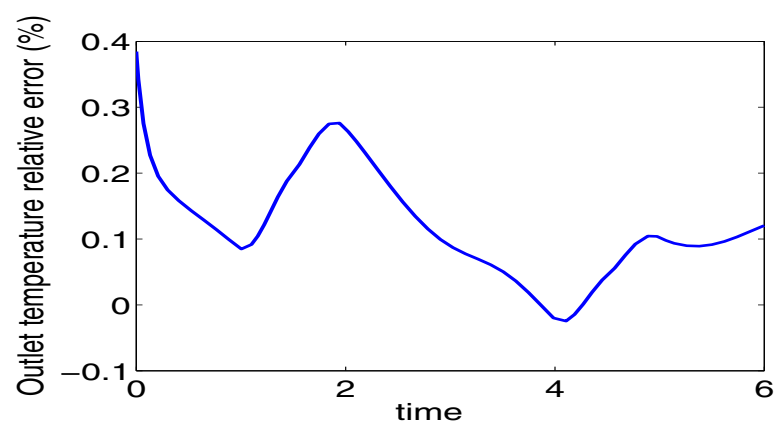

Fig. 5: Outlet temperature relative tracking error

\section{CONCLUSION}

In this paper a new control strategy based on an internal energy-like principle and using a state observer to reconstruct the distributed temperature profile was introduced. Provided that the control input is strictly positive the state observer is exponentially convergent which allows to design an effective control law able to drive the tracking error point-wisely towards zero. Thus, the feedback controller gain and the state observer can be designed separately. In this paper, we have also seen that an appropriate choice of the initial condition of the target model that allows the control law to satisfy the volumetric flow constraints and the pointwise convergence (condition (7) and Remark 3.1). Simulation results have shown the performance of the proposed control law. It is noteworthy that this control method works fine for normal operation mode. In our future work, we will extend our developed control approach to larger process operating modes (startup and shutdown) and more advanced strategies using hierarchical approach to build the reference model will be integrated in the control law. Robust and optimal control methods to deal with the disturbances (the solar radiation and the boundary temperature profile) will also be investigated with more simulations and experimental results

\section{REFERENCES}

[1] E. F. Camacho, M. Berenguel, F. Rubio, and D. Martinez, Control of solar enegy systems. Springer-Verlag London Limited, 2012.

[2] E. Camacho, F. Rubio, M. Berenguel, and L. Valenzuela, "A survey on control schemes for distributed solar collector fields. part i: Modeling and basic control approaches," Solar Energy, vol. 81, pp. 1240-1251, 2007.
[3] P. D. Christofides and P. Daoutidis, "Robust control of hyperbolic pde systems," Chemical Engineering Science, vol. 53, no. 1, pp. 85-105, 1998.

[4] E. Zuazua, "Propagation, observation, and control of waves approximated by finite difference methods," SIAM Review, vol. 47, no. 2, pp. $197-243,2005$.

[5] T. A. Johansen and C. Storaa, "Energy-based control of a distributed solar collector field," Automatica, vol. 38, pp. 1191-1199, 2002.

[6] O. M. Aamo, J. Salvesen, and B. A. Foss, "Observer design using boundary injections for pipeline monitoring and leak detection," International Symposium on Advanced Control of Chemical Processes, pp. 53-58, 2006.

[7] A. Smyshlyaev and M. Krstic, "Adaptive boundary control for unstable parabolic pdes-part ii: Estimation-based designs," Automatica, vol. 43, pp. 1543-1556, 2007.

[8] M. Krstic and A. Smyshlyaev, "Adaptive boundary control for unstable parabolic pdes-part i: Lyapunov design," IEEE Transactions on Automatic Control, vol. 53, no. 7, pp. 1575-1591, 2008.

[9] A. Smyshlyaev, Y. Orlov, and M. Krstic, "Adaptive identification of two unstable pdes with boundary sensing and actuation," International Journal of Adaptive Control and Signal Processing, vol. 23, pp. 131149, 2009.

[10] F. D. Meglio, R. Vazquez, and M. Krstic, "Stabilization of a system of $\mathrm{n}+1$ coupled first-order hyperbolic linear pdes with a single boundary input," IEEE Transactions on Automatic Control, vol. 58, no. 12, pp. 3097-3111, 2013.

[11] F. D. Meglio, D. Bresch-Pietri, and U. J. F. Aarsnes, "An adaptive observer for hyperbolic systems with application to under balanced drilling," Proceedings of the 19th IFAC World Congress, pp. 1139111397, 2014

[12] P. D. Christofides, Nonlinear and robust control of PDE systems, C. I. Byrnes, Ed. Springer Science+Business Media New York, 2001.

[13] H. Shang, J. F. Forbes, and M. Guay, "Feedback control of hyperbolic distributed parameter systems," Chemical Engineering Science, vol. 60, pp. 969-980, 2005.

[14] I. Aksikas, A. Fuxman, F. Forbes, and J. Winkin, "Lq control design of a class of hyperbolic pde systems: Application to fixed-bed reactor," Automatica, vol. 45, pp. 1542-1548, 2009.

[15] A. Pazy, Semigroups of linear operators and applications to partial differential equations, S.-V. N. York, Ed., 1983, vol. 44.

[16] M. Renardy and R. C. Rogers, An introduction to partial differential equations. Springer-Verlag New York, 2004.

[17] C. M. Cirre, M. Berenguel, L. Valenzuela, and R. Klempous, "Reference governor optimization and control of a distributed solar collector field," European Journal of Operational Research, vol. 193, pp. 709717, 2009.

[18] C. Z. Xu and G. Sallet, "Exponential stability and transfer functions of processes governed by symmetric hyperbolic systems," SIAM J. Control Optim., vol. 7, pp. 421-442, 2002. 